In this chamber the air, which has been previously condensed and cooled, is allowed to deposit, in the form of snow, the moisture which it can no longer retain owing to the great diminution of temperature due to expansion. George Punter, whose business it is to look after this snow chamber, rendered the most intelligent assistance in preparing the bars of ice, and in conducting the experiments. In this mode of experimentation the great variation of temperature, namely, between $-30^{\circ} \mathrm{C}$., when the engines are stopped in the evening, and $-12^{\circ} \mathrm{C}$., as a maximum when they begin work in the morning is an unavoid. able drawback. Still, I think that the experiments, although they give uniformly negative results, are worth putting on record.

Experiment $\mathrm{I}$, - A cylinder of ice was cast with a diameter of 3 inches. Over it was hung, as in the well-known Bottomley experiment (NATURF, vol. v. p. I85), a wire loaded with a total weight of 5 Ibs. It was left in the freezing-chamber $6 \frac{1}{2}$ hours. No dent was traceable on the surface of the cylinder.

Experiment 2. - With a similar cylinder and wire the load was increased to ro lbs. and the time to 8 hours, with like negative results.

Experiment 3.-With a similar cylinder and wire the load was further increased to $14 \mathrm{lbs}$. and the time to $7 \frac{1}{2}$ hours, with the same result or absence of result. This experiment would seem to show that the ice refused to yield to a pressure of 20 to 30 atmospheres, or probably more, applied in this way and for this time.

Experiment 4. $\rightarrow$ A bar of ice $1 \frac{1}{2}$ inches thick, $2 \frac{1}{2}$ inches wide, and supported on bearers $13 \frac{1}{2}$ inches apart, was left in the chamber from 12 noon on Monday until 12 noon on Saturday. It showed no sign of bending under its own weight.

Experiment 5.-A similar bar similarly supported was weighted in the micldle with $7 \mathrm{lbs}$., and left for the same time. No sign of bending.

Experiment 6.-A similar bar similarly supported was weighted with $18 \mathrm{lbs}$., and left for the same time. There was no bending perceptible to the eye; but, on removing the apparatus, the bar broke with the jar occasioned by setting it down somewhat carelessly, so that no exact measurement was taken.

Experimen! 7.-A bar of the same length and width, but thinner, tapering somewhat from $\frac{7}{8}$ to $\frac{5}{8}$ of an inch in thickness, was weighted with $7 \mathrm{lbs}$., to which, during the last two days, seven additional pounds were added, and left for the same time. No bending by measurement.

Such negative rejults are just what one would expect on theoretical grounds, and as an inference from previous experiments conducted at temperatures nearer the melting-point. But it is well not to rely on theory or on inference where direct experiment is practicable.

The matter, then, would appear to stand at present somewhat thus. The visco ity of ice, due to whatever cause, is(I) At temperatures at and above the melting-point...considerable. (2) ", ", below but near ,"

What seems now to be wanted is an experimental determination of the lower temperature-limit of viscosity, which would appear to lie somewhere between $-12^{\circ} \mathrm{C}$. and $-3^{\circ} \cdot 5 \mathrm{C}$., but probably nearer the latter temperature.

University College, Bristol

C. Lloyd MORgan

\section{BEN NEVIS}

$\mathrm{A}^{\mathrm{T}}$ the meeting of the Royal Society of Edinburgh held on Monday last, Mr. John Murray, Vice-President, in the chair, Mr. R. T. Omond, Superintendent of the Meteorological Observatory on Ben Nevis, delivered, at "the request of the Council, an address on two years' residence and work there. Mr. Omond, at the outset, recalled the advantages which Ben Nevis presented as a high-level meteorological station, the services of Mr. Clement S. Wragge, and the chief steps that led up to the erection and equipment of the existing permanent observatory. Glancing at some of their daily experiences during last summer and autumn, he mentioned that some 3000 or 4000 tourists climbed the mountain-sometimes at least Ioo in a single afternoon. Since the middle of October, however, not more than half a dozen strangers had ventured up. Some came for information; others were disappointed at finding they could not be fed as well as sheltered; others came to spend the night, but were disappointed at finding they could not do so. Most of the visitors, however, were satisfied, though a little astonished, by the explanation that the building on Ben Nevis was primarily a scientific observatory, and not a hotel. Storms of exceptional and terrific violence were described. Beautiful optical phenomena that had been witnessed, and the comparative scarcity of animal life on the mountain, were next alluded to. Rainbows are seldom seen. Thunderstorms are very rare. The temperatures during winter are not so low as many people think-10 $\mathrm{F}$. is about the lowest recorded as yet, and the ordinary wister temperatures ran from $15^{\circ}$ to $25^{\circ}$. Observing that much must yet be done in the work of the discussion and interpretation of the observations made on Ben Nevis, hefore the observations could be safely used, he proceeded to state some of the more interesting points which Mr. Buchan had already succeeded in approximately establishing: (I) The normal or average temperature and barometric pressure for each month, and the normal differences between these averages and those at sea-level. (2) The daily variation of temperature and pressure during each month. (3) The daily variation in the average velocity of the wind-this being shown to be greater at night than during the day, exactly the reverse of what holds good at sea-level. (4) Variations in the direction of the winds as compared with those prevalent over Scotland at any given time. A comparison of the Ben Nevis winds with those at low-level stations sometimes shows that both are part of one system, whether cyclonic or anti-cyclonic ; but the direction is almost always different, and in the case of cyclonic storms, coming from the west. The observed differences in direction seem to give an indication as to whether the storm centre is to pass to the north or south of Ben Nevis. If this point can be definitely made out, it will obviously be of immense value in forecasting weather. (5) The hygrometric observations indicate that the atmosphere on the Ben shows that during ordinary weather a state of persistent saturation, usually accompanied by fog or mist, prevails; but occasionally a sulden and extraordinary drought sets in, the temperature rises, and the sky clears, not merely of fog, but often of every vestige of cloud, and at the same time the valleys and lower hills are often shrouded in mist, showing that this dryness coming from above is not able to penetrate right down to the sea-level. The thorough investigation of these phenomena is one of the most important pieces of work connected with the Observatory, and may be expected to throw great light on the question of atmospheric circulation. (7) The rainfall of Ben Nevis is greatly in excess of what several theories of the distri $x-$ tion of rain led them to expect-a result possibly due to the great vertical movements of the atmosphere indicated by the hygrometric indications referred to above. Though there are many high-level stations in different parts of the world, none, perhaps, are so favourably situated as Ben Nevis for the investigation of what he had explained is the present great problem in meteorology, namely, the vertical movements of the atmosphere. If the Scottish Meteorological Society were possessed of sufficient funds to establish a completely-equipped observatory at the foot of Ben Nevis as well as on the summit, he was convinced that the science of meteorology would advance far more in a few years than it would by a generation of ordinary work with low-leve] stations alone.

\section{SUNLIGHT ANI THE EARTH'S $A T M O S P H E R E^{1}$}

THERE is, we may remember, a passage in which Plato inquires what would be the thoughts of a man who, having lived from infancy under the roof of a cavern, where the light outside was inferred only by its shadows, was brought for the first time into the full splendors of the sun.

We may have enjoyed the metaphor without thinking that it has any physical application to ourselves who appear to have no roof over our heads, and to see the sun's face daily; while the fact is that if we do not see that we have a roof over our heads in our atmosphere, and do not think of it as one, it is because it seems so transparent and colourless.

Now, I wish to ask your attention to-night to considerations in some degree novel, which appear to me to show that it is not transparent as it appears, and that this seeming colourlessness is a sort of delusion of our senses, owing to which we have never

${ }^{x}$ Lecture delivered at the Royal Institution, April ${ }_{17}$, I885, by S.P. Langley. Communicated by the author. 
in all our lives seen the true colour of the sun, which is in reality blue rather than white, as it luoks, so that this air all about and above us is acting like a coloured glass roof over our heads, or a sort of optical sieve, holding back the excess of blue in the original sunlight, and letting only the white sift down to us.

I will first ask you, then, to consider that this seeming colourlessness of the air may be a delusion of our senses, due to habit, which bas never given us anytling else to compare it with.

If that cave had been lit by sunshine coming through a reddish glass in its roof, would the perpetual dweller in it ever have had an idea but that the sun was red? How is he to know that the glass is "coloured" if he has never in his life anything to compare it with? How can he have any iclea but that this is the sum of all the sun's radiations (corresponding to our idea of whitc or colourless light) ; will not the habit of his life confirm him in the idea that the sun is red; and will he iot think that there is no colour in the glass so long as he cannot go outside to see? Has this any suggestion for us, who have none of us ever been outside our crystal roof to see?

We must all acknowledge in the abstract, that habit is equally s... ong in us whether we dwell in a cave or under the sky, that what we have thought from infancy will probably appear the sole possible explanation, and that, if we want to break its chain, we should put ourselves, at least in imagination, under conditions where it no longer binds us.

The Challenger has dredged from the bottom of the ocean fishes which live habitually at great depths, and whose enormous eyes tell of the correspondingly faint light which must have descended to them through the seemingly transparent water. I will not be as futile a speculation as it may at first seem, to put ourselves in imagination in the condition of creatures under the sea, and ask what the sun may appear to be to them; for if the fish who had never risen above the ocean flor were an intelligent being, might he not plausibly reason that the dim greenish light of his heaven-which is all he has ever knownwas the full splendour of the sun, shining through a medium which all his experience shows is transparent?

We ourselves are, in very fact, living at the floor of a great aërial sea, svhose billows roll hundreds of miles above our heads. Is it not at any rate conceivable that we may have been led into a like fallacy from judging only by what we see at the bottom ? May we not, that is, have been led into the fallacy of assuming that the intervening medium above us is colourless because the light which comes through it is so?

I freely adnit that all men, educated or ignorant, appear to have the evidence of their senses that the air is colourless, and that pure sunlight is white, so that if I venture to ask you to listen to considerations which have lately been bronght forward to show that it is the sun which is blue, and the air rcally acts like an orange veil or like a sieve which picks out the blue and leaves the white, I do so in the confidence that I may appeal to you on other grounds than those I could submit to the primitive man who has his senses alone to trust to ; for the tclucated intelligence possesses those senses equally, and in addition the ability to interpret them by the light of reason, and before this audience it is to that interpretation that I address mysclf.

Permit me a material illustration. You see through this glass, which may typify the intervening medium of air or water, a circle of white light, which may represent the enfeebled disk of the sun when so vicwed. Is this intervening glass coloured or not? It seems nearly colourless; but have we any right to conclude that it is so because it seems so? Are we not taking it for granted that the original light which we see through it is $\mathrm{u}$ hite, and that the glass is colourless, because the light seems unaltered, and is not an appeal to be made here from sence to reason, which, in the educated observer, recalls that white light is made of various colours, and that whether the original light is really white and the glass transparent, or the glass really coloured and so making the white, is to be decided only by experiment, by taking away the possibly deceptive medium? I can take away this glass, which was not colourless, but of a deep orange, and you see that the original light was not white, but intensely blue. If we could take the atmosphere away between us ald the sun, how can we say that the same result might not follow? 'To make the meaning of our illustration clearer, observe that this blueness is not a pure spectral blue. It has in it red, yellow, blue, and all the colours which make up white, but blue in superabundance; so that, though the white is, so to ray, latent there, the dominant effect is blue. The glass coloured veil does not put anything in, but acts I repeat like a sieve straining out the blue, and letting throngh to us the white light which was there in the bluishness, and so may not our air do so too?

I think we already begin to see that it is at any rate conceivable that we may haye been hitherto under a delusion about the true colour of the sun, though of course this is not proving that we have been so, and it will at any rate, I hope, be evident that here is a question raised which ought to be sctlled, for the blueness of the sun, if proven, evidently affects our present knowledge in many ways, and will modify our present views in optics, in meteorology, and in numerous other things. In optics, because we should find that white light is not the sum of the sun's radiations, but only of those dregs of them which have filtered down to us; in meteorology because it is suggested that the temperature of the globe and the condition of man on it, depend in part on a curious selective action of our air, which picks out parts of the solar heat (for instance, that connected with its blue lioht), and holds them back, letting other selected portions come to us, and so altering the conditions on which this heat by which we live, depends; in other ways, innumerable, because, as we know, the sun's heat and light are facts of such central importance, that they affect almost every part of scientific knowledge.

It may be as! ed what :uggested the idea that the sun may be blue rather than any other colour.

My own attention was first directed this way many years ago when measuring the heat and light from different parts of the sun's disk. It is known that the sun has an atmosphere of its own which tempers its heat, and, by cutting off certain radiations and not others, produces the spectral lines we are all familiar with. These lines we customarily study in connection with the ahsorbing vapours of sodium, iron, and so forth, which produce them ; but my own att ntion was particularly given to the regions of absorption, or to the colour it caused, and I found that the sun's body must be deeply bluish, and that it would shed blue light except for this apparently colourless solar atmosphere, which really plays the part of a reddish veil, lettins a little of the blue appear on the centre of the sun's disk where it is thinnest, and staining the edge red, so that to delicate tests the centre of the sun is a pale aqua-marine, and its edge a garnet. The effect I found to be so important, that if this all but invisible solar atmosphere were diminished by but a third part, the temperature of the British islands would rise above that of the torrid zone, and this directed my attention to the great practical impurtance of studying the action of our own terrestrial atmosphere on the sun, and the antecedent probability that our own air was also and indepenciently making the really blue sun into an apparently white one. We actually know then, beyond conjecture, by a comparison of the sun's atmosphere, where it is thickest, and where it is thinnest, that an apparently colourless atmosphere can have such an effect, and analogous observations which I have carried on for many years, but do not now detail, show that the atmosphere of our own planet, this seemingly clear air in which we exist like creatures at the bottom of the sea, does do so.

We look up through our own air as through something so limpid in its purity that it appears scarcely matter at all, and we are apt to forget the enormous mass of what seems of such lightness, but which really presses with nearly a ton to each square foot, so that the weight of all the buildings in this great city, for instance, is less than that of the air above them.

I hope to sbortly describe the method of proof that it too has been acting like an optical sieve, holding back the blue; but it may naturally be asked, "Can our senses have so entirely deceived us that they give no hint of this truth, if it be one? is the appeal wholly to recondite scientific methods, and are there no indications, at least, which we may gather for ourselves?" I think there are, even to our unaided eyes, ind cations that the seemingly transparent air really acts as an orange medium, and keeps the blue light Lack in the upper sky.

If I hold this piece of glass before my eyes, it seems colourless and transparent, but it is proved not to be so by looking through it edgewise, when the light, by traversing a greater extent, brings out its true colour, which is yellow. Every one knows this in every-day cxperience. We shall not get the colour of the ocean by looking at it in a wine-glass, but by gazing through a great depth of it; and so it is with the air. If we look directly up, we look through where it is thinnest; but if we look horizontally through it towards the horizon, through great thicknesses, as at sunrise or sunset, is it not true that this air, where 
we see its real colour most plainly, makes the sun look very plainly yellow or orange?

We not only see here, in humid English skies, the "orange sunset waning slow," but most of us in these days of travel can perfectly testify that the clearest heavens the earth affords, the ro:y tint on the snows of Mont Blanc, forerunning the dawn, or the warm glow of the sun as he sets in Egyptian skies, show this most clearly - show that the atmosphere holds back the blue rays by preference, and lets the orange through.

If, next, we ask, "What has become of the blue that it has stopped?" does not that very blue of the midday sky relate the rest of the story - that blue which Prof. Tyndall has told us is due to the presence of innumerable fine particles in the air, which act selectively on the solar waves, diffusing the blue light towards us? I hope it will be understood that Prof. Tyndall is in no way responsible for my own inferences; but I think it is safe at least to say that the sky is not self-luminous, and that, since it can only be shini $g$ blue at the expense of the sun, all the light this sky sends us has been taken by our atmosphere away from the direct solar beam, which would grow both brighter and bluer if this were restored to it.

If all that has been said so far renders it possible that the sun may be blue, you will still have a right to say that "possibilities" and "maybes" are not evidence, and that no chain of mere hypotheses will draw truth out of her well. We are all of one mind here, and I desire next to call your attention to what I think is evidence.

Remembering that the case of our supposed dweller in the cave who could not get out:ide, or that of the inhabitants of the ocean-floor who cannot rise to the surface, is really like our own, over whose heads is a crystalline roof which no man from the beginning of time has ever got outside of, an upper sea to whose surface we have never risen; we recognise that if we could rise to the surface, leaving the medium whose effect is in dispute wholly beneath us, we should see the sun as it is, and get proof of an incontrovertible kind; and that, if we cannot entirely do this, we shall get nearest to proof under our real circumstances by going as high as we : can in a balloon, or by ascending a very high mountain. The balloon will not do, because we have to use heavy apparatus requiring a solid foundation. The proof to which I ask your kind attention, then, is that derived from the actual ascent of a remarkable mountain by an expedition undertaken for that purpose, which carried a whole physical laboratory up to a point where nearly one-half the whole atmosphere lay below us. I wish to describe the difference we found in the sun's energy at the bottom of the mountain and at the top, and then the means we took to allow for the effect of that part of the earth's atmosphere still over our heads even here, so that we may be said to have virtually got outside it altogether.

Before we begin our ascent, let me explain more clearly what we are going to seek. We need not expect to find that the original sunlight is a pure monochromatic blue by any means, but that though its rays contain red, orange, blue, and all the other spectral colours, the blue, the violet and the allied tints were originally there in disproportionate amounts, so that, though all which make white were present from the first, the refrangible end of the spectrum had such an excess of colour that the dominant effect was that of a blueish sun. In the same way, when I say briefly that our atmosphere has absorbed this excess of blue and let the white reach us, I mean, more strictly speaking, that this atmosphere has absorbed all the colours, but, selectively, taking out more orange than red, more green than orange, more blue than green ; so that its action is wh lly a taking out-an action like that which you now see roing on with this sieve, sifting a mixture of blue and white beads, and holding back the blue while letting the white fal down

This experiment only rudely typifies the action of the atmosphere, which is di-criminating and selective in an amazing degree, and as there are really an infinite number of shades of colour in the spectrum, it would take for ever to describe the action in detail. It is merely for brevity, then, that we now unite the more refrangible colours under the general word "blue," and the others under the corresponding terms "orange" or "red."

All that I have the honour to lay before you, is less an announcement of absol ite novelty than an appeal to your already acquired knowledge and to your reasion as superior to the delusions of sense. I have, then, no novel experiment to offer, but to ask you to look at some familiar ones in a new light.
We are most of us familiar, for instance, with that devised by Sir Isaac Newton to show that white light is compounded of blue, red, and other colours, where, by turning a coloured wheel rapidly, all blend into a grayish white. Here you see the "seven colours" on the screen; but. though all are here, I have intentionally arranged them, so that there is too much blue, and the combined result is a very bluish white which may roughly stand for that of the original sun-ray. I now alter the proportion of the colours so as to virtually take out the excess of blue, and the result is colour'ess or white light. White, then, is not necessarily made by combining the "seven colours," or any number of them, unless they are there in just proportion (which is in effect what Newton himself says) ; and white, then, may he made out of such a bluish light as we have described, not by putting anything to it, but by taking away the excess which is there already.

Here, again, are two sectors-one blue, one orange-yellow with the blue in excess, making a bluish disk where they are revolved. I take out the excess of blue, and now what remains is white.

Here is the spectrum itself on the screen, but a spectrum which has been artificially modified so that the blue end is relatively too strong. I recombine the colours (by Prof. Rood's ingenious device of an elastic mirror), and they do not make a pure white, but one tinted with blue. I take out the original excess of blue, and what remains combines into a pure white. Please bear in mind that when we "put in "blue here, we have to do so by straining out other light through some obscuring medium, which makes the spectrum darker; but that, in the case of the actual sunlight, introducing more blue, introduces more light, and makes the spectrum brighter.

The spectrum on the screen ought to be male still brighter in the blue than it is--far, far brighter-and then it might represent to us the original solar spectrum before it has suffered any absorption either in the sun's atmosphere or our own. The Frauenhofer lines do not appear in it, for these, when found in the solar spectrum, show that certain individual rays have been stopped, or selected for a bsorption by the intervening atmo spheres; and though even the few yards of atmosphere between the lamp and the screen absorb, it is not enough to show.

Our spectrum, as it appears before absorption, might be compared to an army divided into numerous brigades, each wearing a distinct uniform, one red, one green, one blue, so that all the colours are represented eazh by its own body. If, to represent the light absorbed as it progresses, we supposed that the army advances under a fire which thins its numbers, we should have to consider that (to give the case of nature) this destructive fire was directed chiefly against those divisions which were dre ssed in blue, or allied colours, so that the army was thinned out unequally, many men in blue being killed off for one in red, and that by the time it has advanced a certain distance under fire the proportion of the men in each brigade has been altered, the red being comparatively unhurt. Almost all absorption is thus selective in its action, and often in an astoni-hing degree, killing off, so to speak, certain rays in preference to others, as though by an intelligent choice, and destroying most, not only of certain divisions (to continue our illustration), but even picking out certain files in each company. Every ray, then, has its own individuality, and on this I cannot too strongly insist ; for just as two men retain their personalities under the same red uniform, and one may fall and the other survive, though they touch shoulders in the ranks, so in the spectrum certain parts will be blotted out by absorption; while others next to them may escape.

To illustrate this selective absorpti in, I put a piece of didymium glass in the path of the ray. It will, of course, ab orb some of the light, but instead of dimming the whole spectrum, we might almost say it his arbitrarily chosen to select one narrow part for action, in this particular case choosing a narrow file near the orange, and letting all the rest go unharmed. In this arbitrary way o:ur atmo phere operates, but in a far more complex manner, taking out a narrow file here and another there, in hundreds of places, all through the spectrum, but on the whole much the most in the blue, the Frauenhofer lines being merely part of the evidence of this wonderful quasi-intellisent action which bears the name of selective absorption.

Before we leave this spectrum, let us recall on most important matter. We know that here beyond the red is solar energy in the form of heat which we cannot see, but not on that account any less important. More than half the whole power of the 
sun is here invisible, and if we are to study completely the action of our atmosphere, we shall have to pay great attention to this part, and find out some way of determining the loss in it, which will be difficult, for the ultra-red end is not only invisible, but compressed, the red end being shut up like the closed pages of a book, as you may notice by comparing the narrowness of the red with the width of the blue.

Now refraction by a prism is not the only way of forming a spectrum. Nature furnishes us colour not only from the rainbow, but from non-transparent substances like mother-of-pearl, where the iridescent hues are due to microscopically fine lines. Art has lately surpassed nature in these wonderful "gratings," consisting of pieces of polished metal, in which we see at first nothing to account for the splendid play of colour apperently pouring out from them like light from an opal, hut which, on examination with a powerful microscope, show lines so narrow that there are from 50 to 100 in the thickness of a fine human hair, and all spaced with wonderful precision.

This grating is equal in defining power to many such prisms as we have just been looking at, but its light does not show well upon the screen. You will see, however, that its spectrum differs from that of the prism, in that in this case the red end is expanded, as compared with the violet, and the invisible ultrared is expanded still more, so that this will be the best means for us to use in exploring that "dark continent" of invisible heat found not only in the spectrum of the sun, but of the electric light, and of all incandescent bodies, and of whose existence we already know from Herschel and Tyndall.

Now we cannot reproduce the actual solar spectrum on the screen without the sun itself, but here are photographs of it, which show parts of the losses the different colours have suffered on their way to us. We have before us the well-known Frauenhofer lines, due, you remember, not only to absorption in the sun's atmosphere, but also to absorption in our own. We have been used to think of them in connection with their cause, one being due to the absorption of iron-vapour in the sun, another to that of water-vapour in our own air, and so forth; but now I ask you to think of them only in connection with the fact that each is due to the absorption of some part of the original light, and that collectively they tell much of the story of what has happened to that light on its way down to us. Observe, for instance, how much thicker they lie in the blue end than in the red-another evidence of the great proportionate loss in the blue.

If we could restore all the lost light in these lines, we should get back partly to the original condition of things at the very fount, and, so far as our own air is concerned, that is what we are to ascend the mountain for-to see, by going up through nearly half of the atmosphere, what the rate of loss is in each ray by actual trial ; then, knowing this rate, to be able to allow for the loss in the other part still above the mountain-top, and, finally, by recombining these rays to get the loss as a whole. Remember, however, always, that the most important part of the solar energy is in the dark spectrum which we do not see, but which, if we could see, we should probably find to have numernus absorption-spaces in it corresponding to the Franenhofer lines, but where heat has been stopped out rather than light. To make our research thorough, then, we ought not to trust to the eye only, or even chiefly, but have some way of investigating the whole spectrum; the invisible in which the sun's power chiefly lies, as well as the visible, and both with an instrument that would discriminate the energy in these very narrow spaces, like an eye to see in the dark; and if science possesses no such instrument, then it may be necessary to invent one.

The linear thermopile is nearest to it of any, and we all here know what good work it has done, but even that is not sensitive enough to measure in the grating spectrum, in some parts of which the heat is 400 times weaker than in that of a prism, and we want to observe this invisible heat in very narrow spaces. Something like this has been provided since by Capt. Abney's most valuable researches, but these did not at the time go low enough f $\sim r$ my purpose, and I spent nearly a year before ascending the mountain in inventing and perfecting the new instrument for measuring these, which I have called the "bolometer" or "ray-measurer." The principle on which it is founded is the same as that employed by my late lamented friend, Sir Wm. Siemens, for measuring temperatures at the bottom of the sea, which is that a smaller electric current flows through a warm wire than through a cold one.

One great difficulty was to make the conducting wire very thin, and yet continuous, and for this purpose almost endless experiments were made, among other substances pure gold having been obtained by chemical means in a plate so thin that it transmitted a sea-green light through the solid substance of the metal. This proving unsuitable, I learned that iron had been rolled of extraordinary thinness in a contest of skill between some English and American iron-masters, and, procuring some, I found that 15,000 of the iron plates they had rolled, laid one on the other, would make but one English inch. Here is some of it, rolled between the same rolls which turn out plates for an iron-clad, but so thin that, as I let it drop, the iron plate flutters down like a dead leaf. Out of this the first bolometers were made, and I may mention that the cost of these earlier experiments was met from a legacy by the founder of the Royal Institution, Count Rumford. The iron is now replaced by platinum, in wires or rasher tapes, from $I-2000$ to $I-20$, 00oth of an inch thick, one of which is within this button, where it is all but invisible, being far finer than a human hair. I will project it on the screen, placing a common small pin beside it as a standard of comparison. This button is placed in this ebonite case, and the thread is moved by this micrometer screw, by which it can be set like the spider line of a reticule; but by means of this cable, connecting it to the galvanometer, this thread acts as though sensitive, like a nerve laid bare to every indication of heat and cold. It is then a sort of sentient thing: what the eye sees as light it feels as heat, and what the eye sees as a narrow band of darkness (the Frauenhofer line) this feels as a narrow belt of cold, so that when moved parallel to itself and the Fratenhofer lines down the spectrum it registers their presence.

It is true we can see these in the visible spectrum, but you remember we propose to explore the invisible also, and since to this the dark is the same as the light, it will feel absorption lines in the infra-red which might remain otherwise unknown.

I have spent a long time in these preliminary researches; in indirect methods for determining the absorption of our atmosphere, and in experiments and calculations which I do not detail, but it is so often supposed that scientific investigation is a sort of happy guessing, and so little is realised of the labour of preparation and proof, that I have been somewhat particular in describing the essential parts of the apparatus finally employed, and now we must pass to the scene of their use.

(To be continued.)

\section{THE INSTITUTION OF MECHANICAL ENGINEERS}

A VERY interesting discussion on the merits of the Maxim automatic machine-gun, which was described in NATURE, vol. xxxi. p. 4I4, took place at the special meeting of the Institution held on the 3oth ult. In reply to Mr. Carbutt, M.P., the inventor explained that the recoil of the gun, which is utilised for loading and firing, did not interfere with the accuracy of aim, and instanced the circumstance that as good target-practice was made in firing from the shotlder as with fixed rifles; whilst the energy of recoil was sufficient to carry on the automatic action, whether the muzzle of the gun was elevated or depressed. As regarded keeping the barrel cool, he found that the waterjacket around the body of the gun acted most efficiently, as gunpowder in exploding produced very little heat-energy, or, as he put it, "he should not buy gunpowder to raise steam." During all his experiments he had used only Government cartridges, and had never found one to fail; he thought it would be an advantage if cartridges were packed in cases containing calcium chloride or other hydroscopic material, so that they might not be injured by moisture.

The gun was frequently fired during the meeting, and its automatic action was thoroughly shown, as well as its freedom from danger should a cartridge hang fire. Mr. Maxim had a most enthusiastic reception, the general feeling of the speakers and of the meeting being in favour of the gun being taken up by the British Government, the President and Mr. Adamson giving it as their opinion that, if the necessity should occur, 1000 of these guns could be produced weekly at a month's notice, when their use night have as material an effect on a campaign as the needle-gun had at Sadowa. Mr. Maxim is now experimenting with a more recent form of gun of his invention, which fires a projectile $3 \mathrm{lbs}$. weight at the rate of 120 shots a minute; in this the cartridges are fed from above, which much simplifies the mechanical arrangement, as no apparatus has to 